\section{Nature's way}

\section{Antonia J. Jones}

Genetic Programming: On the Programming of Computers by Means of Natural Selection. By John R. Koza. MIT Press: 1992. Pp. 819. \$55, £49.50.

IN 1975, John Holland published his classic book on adaptation in natural and artificial systems. His idea was that evolution by means of natural selection can be simulated on a computer and used to optimize the design of any given structure. His work differed from earlier attempts in two important respects. First, he capitalized on the idea that sexual reproduction is a critical accelerator of the evolutionary process and built this feature into his genetic algorithms using a 'crossover' operator that mixes the genes of both parents. Second, he gave genetic algorithms a theoretical credibility by showing mathematically why they could be expected to work better than, say, a random search.

It was inevitable that these ideas would eventually be applied directly to breed programs themselves, and Koza's book is the first substantial step in this direction. Holland's theory does not apply directly to Koza's scheme and Koza does not attempt to erect a theoretical edifice; he seeks only to demonstrate that the method does, in some sense, work. Major software systems are the most complex structures we have ever sought to engineer, and their reliability is often crucial. Consequently, formal specification and proof of correctness are important. The idea of a serious scientific study devoted to producing formally incorrect programs may therefore seem like heresy. Nonetheless, that is exactly what Koza and his students are doing and the results are rather amazing.

Obviously, if one simply splices bits of code together from two programs the chances that the resulting structure will even run, let alone do anything useful, are vanishingly small. Koza overcomes this difficulty by working in LISP, in which the program is essentially its own parse tree (a structure that demonstrates how a given, syntactically correct sentence may be derived from the rules of the underlying grammar). So a crossover that grafts a subtree from one program into another will at least produce a syntactically correct child. Of course, this is a convenience and not a restriction of generality. Having created a child program using crossover, the result is then run, once or many times, so that its fitness (as defined by criteria laid down at the start) can be automatically judged. The fitness is used to calculate the probability that the program will be a parent

in the next generation.

The criterion of fitness is analogous to a specification, and Koza chooses example problems for which this criterion is relatively easy to write down. Some are classic test problems in artificial intelligence, such as cart centring, broom balancing, truck reversing and box stacking. Others involve a pursuerevader game, the control of a two-link robot arm, emergent collective behaviour of ants, task prioritizing (how to play Pac-Man) and the spontaneous emergence (in a nontrivial way) of self-replicating and self-improving programs. The 'Genetic Programming Paradigm' solves them all successfully, in the sense that workable, reliable programs emerge fairly rapidly.

To argue that it is hard to imagine the method being used to produce (say) a

\title{
Aqueous surface reactions
}

\section{William H. Casey}

Chemistry of the Solid-Water Interface: Processes at the Mineral-Water and Particle-Water Interface in Natural Systems. By Werner Stumm. Wiley: 1992. Pp. 428. £34.50, \$48.95 (pbk).

AQueous geochemistry is undergoing an upheaval forced, in part, by increasing pressure on governments to assess the safety of waste repositories and the fate of agricultural chemicals. For many years, processes taking less than $10^{3}$ years or so were considered breathlessly fast. Scientific emphasis was placed on thermodynamic calculations that elucidated possible, rather than actual, pathways in the chemical history of the Earth. The length and timescales were simply too vast for close testing of the predictions.

The focus is now changed. Predictions about contaminant migration in the Earth are regularly tested but generally fail. The mathematics is daunting because sets of nonlinear equations describing chemical equilibria must be solved simultaneously with the equations of groundwater flow. Emphasis has been placed on ad hoc simplifications that reduce the mathematics to a linear form (but the chemistry to near absurdity). These simplifications work best when they describe mineral-fluid surface reactions, which are usually rapid and extensive.

Geochemists have established a nascent cottage industry adapting Werner Stumm and James Morgan's Aquatic Chemistry (Wiley, 1981) for use as a guide to these low-temperature processes. This is a wonderful monograph with a page or two devoted to spreadsheet is to miss the point. Producing software in cases where the rules that map input to output are precisely known is much less of a problem than in cases where the circumstances faced by the software are hard to foresee and the rules for dealing with these circumstances are largely unknown. Many of the central problems in artificial intelligence fall into this category and Koza's scheme offers an intriguing new possibility for automatic programming. The book is a tour de force of carefully presented case studies and includes LISP code with which the doubtful can verify the author's results.

Antonia J. Jones is in the Department of Computing, Imperial College of Science, Technology and Medicine, Exhibition Road, London SW7 2BT, UK. each aspect of natural water chemistry. But it is comprehensive to the point of being monolithic (780 pages): undergraduate students take one look and scurry off to study materials science.

In Chemistry of the Solid-Water Interface, Stumm expands the discussion of aqueous surface chemistry. He takes the familiar approach of treating surfaces as assemblages of coordinative functional groups. The chemistry of aqueous-metal complexes provides a key to understanding reactions at these functional groups.

The real strength of the new book, as in Aquatic Chemistry, is its breadth. The author goes well beyond simple reactions at oxide mineral surfaces to embrace hydrophobicity, polyelectrolytes and simple models of contaminant migration. Even bacterial adhesion is covered. Such discussion is timely: the attention of geochemists is increasingly drawn to environments that are contaminated with solvents and halogenated hydrocarbons and away from purely inorganic systems.

The book is, however, more a review of the author's interests and collaborations than an integrated text. This narrow focus is perhaps forgivable, as the book marks the culmination of Stumm's fruitful career as director of the Swiss Federal Institute of Technology. One need only ponder Carrick Eggleston's magnificent cover image of atomic steps on goethite (an iron oxide mineral) in situ to realize that extraordinary advances punctuate our progress in understanding natural water chemistry.

William H. Casey is in the Department of Land, Air and Water Resources, University of California, Davis, California 956168627, USA. 